\title{
A concept of precise kinematic positioning and flight-state monitoring from the AGMASCO practice
}

\author{
Guochang $\mathrm{Xu}$ \\ GeoForschungsZentrum Potsdam (GFZ), Division Kinematics and Dynamics of the Earth, Telegrafenberg, 14473 Potsdam, Germany
}

(Received November 30, 1999; Revised June 8, 2000; Accepted June 8, 2000)

\begin{abstract}
A concept of precise kinematic positioning and flight-state monitoring of an airborne remote sensing system is presented here, based on practical experiences from the EU project AGMASCO. Within the project about two months of kinematic GPS flight data and static reference data have been collected in Europe over four campaigns during the past three years. An independently developed GPS software package as well as several commercial GPS software packages have been used for data processing. In this paper, the methods of creating the tropospheric model for the aircraft trajectory and the use of static ambiguity results as conditions in the kinematic positioning are discussed. These concepts are implemented in the kinematic GPS software KSGSoft, and have demonstrated excellent performance.
\end{abstract}

\section{Introduction}

The EU (European Union) project AGMASCO (Airborne Geoid Mapping System for Coastal Oceanography), in which five European institutions participated, has collected about two months of static and airborne kinematic GPS data for the purpose of kinematic positioning and flight-state monitoring of an airborne remote sensing system. The remote sensing system includes an aerogravimeter, accelerometer, radar and laser altimeter, INS and data logger. During the past three years, four flight campaigns were performed in Europe. They are the test campaign in Braunschweig in June 1996, the Skagerrak campaign in September 1996 (Fig. 1), the Fram Strait campaign in July 1997 and the Azores campaign in October 1997 (Fig. 2). Two to three kinematic GPS antennas were mounted on the fuselage, the back and the wing of the aircraft, and at least three GPS receivers were used as static reference receivers.

The above mentioned remote sensing system has two important objectives: measuring the gravity acceleration of the Earth and determining the sea surface topography. Because the aerogravimeter (or accelerometer) and the altimeter are firmly attached to the aircraft, kinematic positioning and flight-state monitoring using GPS plays a key role for determining the flight acceleration, velocity and position, as well as orientation of the aircraft. The high sensitivity of the sensors requires high quality aircraft positioning and flightstate monitoring. Therefore new strategies and methods have been studied, developed, tested and implemented for the GPS data processing.

The adopted concept of precise kinematic positioning and flight-state monitoring are discussed in Section 2 and 3 respectively.

Copy right $(\mathcal{C}$ The Society of Geomagnetism and Earth, Planetary and Space Sciences (SGEPSS); The Seismological Society of Japan; The Volcanological Society of Japan; The Geodetic Society of Japan; The Japanese Society for Planetary Sciences.

\section{Concept of Precise Kinematic Positioning}

A vast literature exists on the topic of precise kinematic positioning (see, for example, Goad and Remondi, 1984; Wang et al., 1988; Schwarz et al., 1989; Cannon et al., 1997; Hofmann-Wellenhof et al., 1997). Based on the AGMASCO practice a modified concept has been developed and applied to the data processing.

\subsection{Combining the static reference stations with IGS}

It is well known that the differential GPS positioning results depend on the accuracy of the reference station(s). However, it is not quite clear how strong this dependency is, or in other words, how accurately the reference coordinates should be determined for use in kinematic differential positioning. During the AGMASCO data processing it was noticed that the accuracy of the reference coordinates is very important. A bias in the reference station coordinates will cause not only a bias in the kinematic flight path, but also a significant linear trend. Such a linear trend depends on the flight direction and the location of the reference receiver(s). Therefore, in precise kinematic positioning, the coordinates of the static reference stations should be carefully determined by, for example, connecting these stations to the nearby IGS stations. A detailed study of the relationship between the reference station coordinate accuracy and the quality of kinematic and static positioning has been carried out by Jensen (1999).

\subsection{Earth tide and loading tide corrections}

A detailed study of the Earth tide effects on GPS kinematic/static positioning is given in Xu and Knudsen (2000). For airborne kinematic differential GPS positioning, Earth tide effects on the static reference station need to be corrected for. Such tidal effects could reach up to $30 \mathrm{~cm}$ in Denmark and Greenland, and $60 \mathrm{~cm}$ at other locations in the world. Tidal effects could induce a 'drift' over a few hours of measurement duration. For ground-based kinematic and static differential GPS positioning with baseline lengths less 


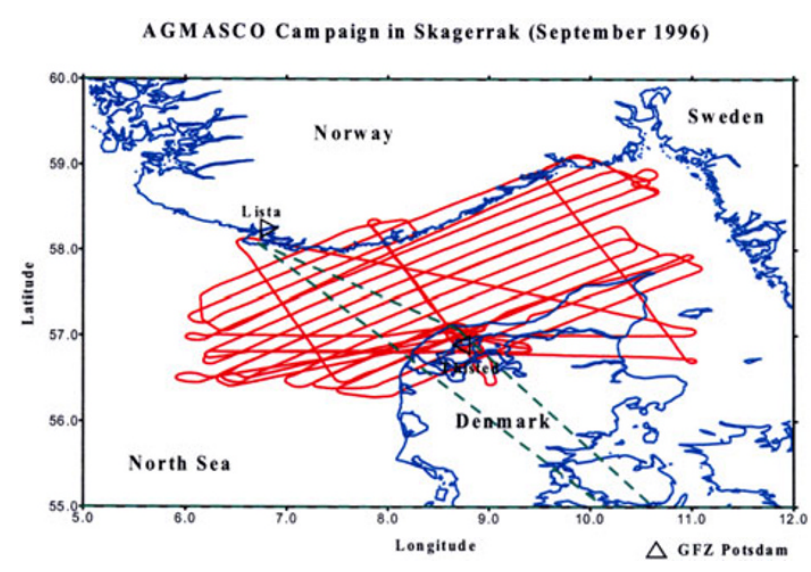

Fig. 1. Flights in the Skagerrak campaign.

than $80 \mathrm{~km}$, the impact of the Earth tide effects could reach more than $5 \mathrm{~mm}$. In precise applications of GPS positioning, both in kinematic and static cases, the Earth tide effects have to therefore be taken into account even for a relatively small local GPS network.

Ocean loading tide effects could also reach up to a few $\mathrm{cm}$ in magnitude, in special cases (Ramatschi, 1998). Generally the ocean loading tide effects should be considered as being at the $\mathrm{cm}$ level in coastal areas, so that these effects have to also be corrected for in GPS data processing. However, unlike the Earth tide, ocean loading tide effects can only be modelled by ocean tide models at about the $60 \%$ to $90 \%$ level (Ramatschi, 1998). Therefore simply using a model to correct for the effects is not enough, and a detailed study of ocean loading tide effects is necessary for precise GPS positioning. It is, however, possible to use GPS for determining the parameters of the local ocean loading tide effects (Shfaqat, 1999).

\subsection{Multiple static reference stations for kinematic po- sitioning}

In differential GPS kinematic positioning usually there is only one static reference receiver used. It is obvious that if multiple static references are used, the reference station dependent errors, such as due to the troposphere and ionosphere as well as ocean loading tide effects, could be reduced and the geometric stability could be strengthened. For simplicity, only the case of using two static reference receivers will be discussed here. In Fig. 3, 1 and 2 denote the static reference receivers, and $k$ the kinematic object. Suppose the two static stations are placed close by and both have the same GPS satellites in view. Using one static reference receiver for kinematic positioning, one has unknown vector $\left[X_{k} N_{1 k}\right]$, where $X$ is the coordinate sub-vector and $N$ is the ambiguity sub-vector. Using two static reference receivers, one has unknown vector $\left[X_{k} N_{1 k} N_{2 k}\right.$, because the unknown coordinate sub-vector $X$ is the same. The number of elements of the sub-vector $N$ compared with that of $X$ is very small in the kinematic case. Therefore, by using multiple static reference receivers for kinematic positioning, the total number of observations is increased, but the total number of unknowns remains almost the same, hence the results will be modified.

Furthermore, according to the definition of the double- differenced ambiguity, one has:

$$
N_{1 k}-N_{2 k}=N_{12},
$$

where $N_{12}$ is the double-difference ambiguity vector of the static baseline, which can be obtained from the static solution. Using Eq. (1), $N_{2 k}$ can be represented by $\left(N_{1 k}-N_{12}\right)$. Thus, by using two static reference receivers for kinematic positioning, one has nearly doubled the number of observables, yet the unknowns remain the same if in addition the static results are used. (Usually the static measurements can be made over a longer time and hence the static results can be obtained precisely.)

Using multiple static reference receivers and introducing the ambiguities from the static solution as conditions, the accuracy of the kinematic positioning can be increased significantly. An example showing the differences in the height of the front antenna determined using multiple reference receivers, with and without using the static ambiguity condition, is given in Fig. 4 (the ambiguity float solutions are used). The average and standard deviation of the differences are 27.07 and $4.34 \mathrm{~cm}$, respectively. These results clearly indicate that the multiple static conditions have modified the results. A change of ambiguity not only caused a bias in the position solution, but also a high frequency variation. The base-base separation is about $200 \mathrm{~km}$ and the length of the kinematic path is about $400 \mathrm{~km}$ (cf. Fig. 1).

For three or more static reference receivers, similar arguments and improved results can be presented.

\subsection{Introducing height information as a condition}

Even after making use of multiple static reference receivers and the static conditions, the ambiguities in the kinematic positioning can still be wrong. In such a case there could be a bias and variation in the kinematic trajectory. Therefore introducing the height information of the aircraft at the start or/and resting point into the data processing is a great help, especially in airborne altimetry applications. The bias of the results obtained by using different software can then be eliminated.

\subsection{Creating a kinematic troposphere model}

Using the multiple static reference receivers, the parameters of the tropospheric model can be determined. Using these parameters, the tropospheric model parameter for the kinematic receiver can be interpolated. Such a model, however, generally is only suitable for the footprint point of the kinematic platform. Therefore the vertical gradient of temperature and the exponential changes of the pressure and humidity (Syndergaard, 1999) are introduced into the standard model to create a tropospheric model for the kinematic station in the air. This is of course not an ideal model, however, it is a reasonable model.

\subsection{Higher order ionospheric effects correction}

For long distance kinematic positioning the ionospherefree combination has to be used to eliminate the ionosphere delay effects. It is well known that the ionosphere-free combination is indeed only a first order approximation (Klobuchar, 1996). The second order ionospheric effect is about $0.1 \%$ of that of the first order (Syndergaard, 1999). Therefore the residual ionospheric effects can reach the few $\mathrm{cm}$ level. This has to be taken into account by some form of modelling of the total ionospheric effects (Xu, 1999b). 


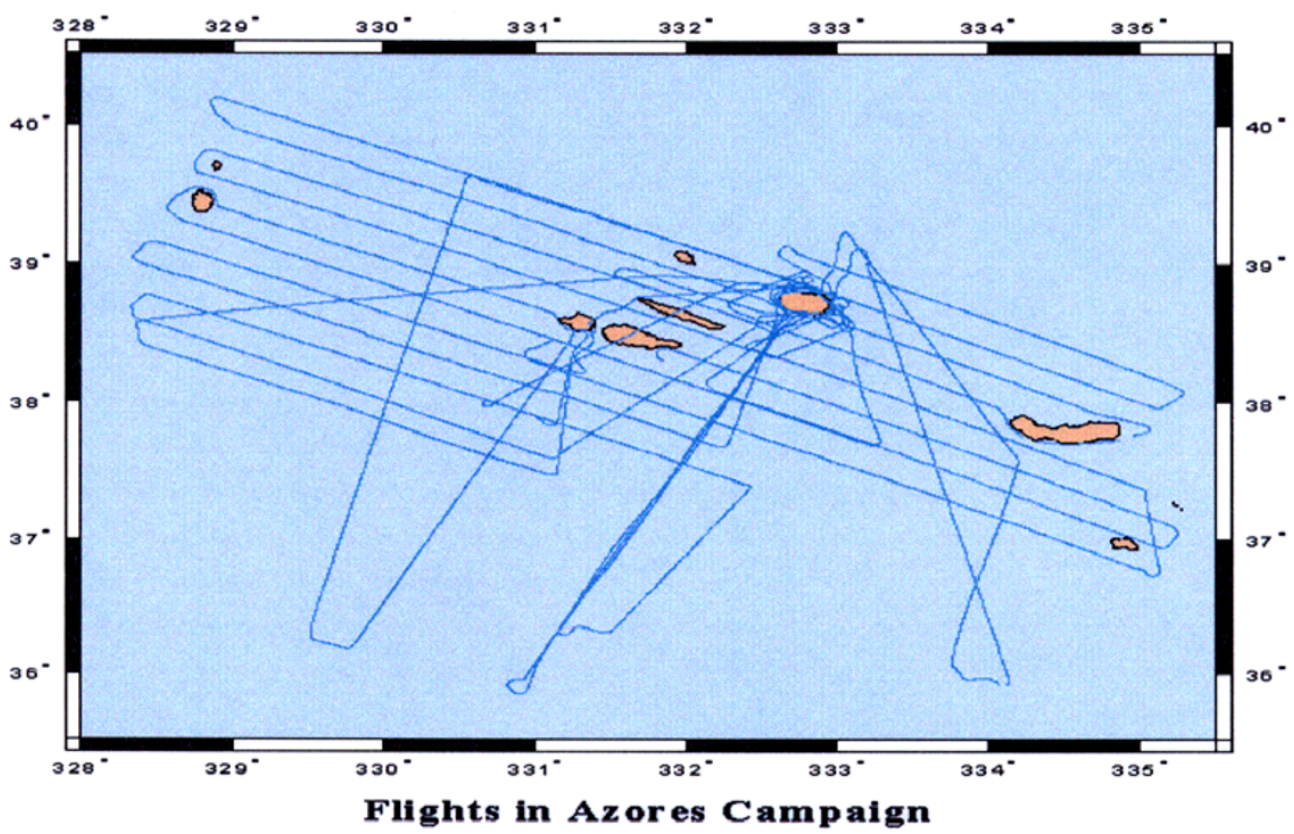

Fig. 2. Flights in the Azores campaign.

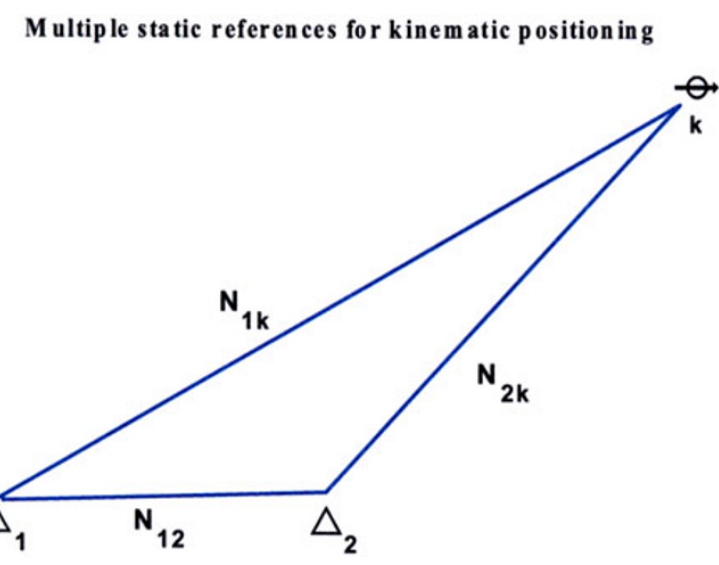

Fig. 3. Usage of multiple static references.

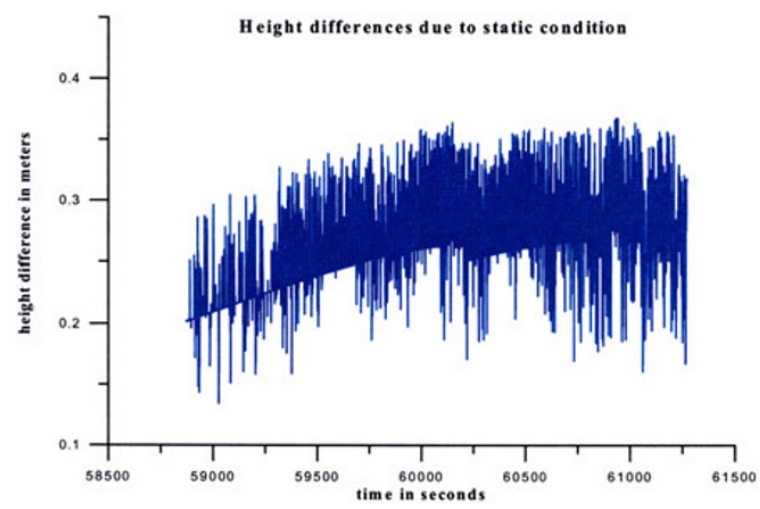

Fig. 4. Height differences with and without ambiguity conditions.

\subsection{A general method of integer ambiguity fixing}

An integer ambiguity search method based on the conditional adjustment theory was proposed in Xu (1999a). This method has been implemented in the GPS software KSGSoft (Kinematic/Static GPS Software), developed at the GFZ Potsdam (Xu et al., 1998), and used extensively for real data processing in this EU project AGMASCO (Xu et al., 1997). The search can be carried out in the coordinate domain, in the ambiguity domain (or both). Most other least squares ambiguity search methods (Euler and Landau, 1992; Teunissen, 1995; Merbart, 1995; Han and Rizos, 1995, 1997) are special cases of this algorithm, if only the ambiguity search domain is selected and without considering the uncertainty of ambiguity. By taking the coordinate and ambiguity residuals into account, a criterion for ambiguity searching is also proposed to ensure an optimal search result. Detailed formulas and their application can be found in Xu (1999a). The exact relationship between the general criterion and the least squares ambiguity search criterion is derived and illustrated by numerical examples in Xu (2000).

\section{Concept of Flight-State Monitoring}

For flight-state monitoring of an aircraft, it is necessary that several GPS antennas be used. To be determined are the relative positions between the multiple antennas. Using as an example the method presented in Section 2, the position and velocity of one of the kinematic antennas could be determined. Using this point as a reference, the related position differences of other antennas can be determined. Because the distances between the multiple antennas are only a few metres, through the differential method the atmospheric effects will be eliminated, and therefore only the single frequency L1 observations are needed for this purpose. In addition, due to the short ranges, such differential positioning can be performed with high accuracy. 
Early stage tests of multiple kinematic GPS antennas mounted on a platform were made for checking purposes, using the known baseline lengths. Typically such checks indicate that the distance has a systematic bias if the distance is computed from the two positions, and these two positions are determined independently. However, a combined solution of the multiple kinematic positioning problem does not overcome the distance bias problem completely because of inaccuracies in the ambiguity solution. Therefore, for precise flight-state monitoring it is necessary to introduce the known distances between the antennas fixed on the aircraft as additional constrains in the data processing.

Because of the short distances, the linearization of the condition cannot be done precisely in the initial step, and therefore an iterative process has to be used. The conditions can be used in a conditional adjustment, or the conditions can be used for eliminating unknowns. Both methods are equivalent.

Flight-state is usually represented by so-called 'state angles' (heading, pitch and roll). They are rotation angles between the body and the local horizontal coordinate frames of the aircraft. The axes of the local horizontal frame are selected as: the $x^{b}$ axis points out the nose, the $y^{b}$ axis points to the right parallel to the wing, and $z^{b}$ axis points out the belly to form a right-handed coordinate system, where $b$ denotes the body frame. The body frame can be rotated to be aligned to the local horizontal frame in a positive, right-handed sense in three steps. First, the body frame is rotated about the local vertical downward axis $z$ by angle $\psi$ (heading). Then the body frame is rotated about the new $y^{b}$ axis by angle $\theta$ (pitch). Finally, the body frame is rotated about the new $x^{b}$ axis by angle $\phi$ (roll). In the local horizontal coordinate system, heading is the azimuth of the axis $x^{b}$ of body frame, pitch is the elevation of the axis $x^{b}$ of the aircraft, roll is the elevation of the axis $y^{b}$ of the aircraft. Note that the directions of the axis $x^{b}$ and the velocity vector of the aircraft are usually not the same. Through kinematic positioning the three flight-state angles can be computed (Cohen, 1996).

Comparisons of numerical GPS flight-state monitoring results are made with the results of an INS. It is possible using GPS to determine the heading with accuracy up to $0.1 \mathrm{de}$ gree, pitch and roll up to 0.2 degree. In our case, the distances between the three antennas were $5.224 \mathrm{~m}, 5.510 \mathrm{~m}, 4.798 \mathrm{~m}$.

\section{Conclusions}

GPS research during the AGMASCO project has concluded that GPS is able to be used for airborne kinematic positioning and flight-state monitoring to fulfil the needs of navigation of a remote sensing system, for applications in aerogravimetry and oceanography.

A methodology has been proposed for precise kinematic GPS positioning that addresses the following issues:

- using IGS stations to obtain precise reference station coordinates, and introducing Earth tide and ocean loading tide corrections,

- introducing multiple static reference receivers, and using the static ambiguity solution as conditions,

- introducing the initial height information as a condition, and introducing the troposphere model to the aircraft kinematic GPS stations, and

- modelling the higher order ionospheric effects, and using a method of ambiguity searching in the coordinate and ambiguity domains.

For flight-state monitoring, the kinematic reference and single frequency L1 are used. Known distances between the multiple kinematic antennas are used as additional constraints. Results have indicated adequate performance of this methodology.

Acknowledgments. This study is carried out within the framework of the EU AGMASCO project supported by the EU MAST Project MAS3-CT95-0014. All colleagues in the AGMASCO team are thanked for their valuable cooperation.

\section{References}

Cannon, M. E., G. Lachapelle, M. Szarmes, J. Herbert, J. Keith, and S. Jokerst, DGPS Kinematic Carrier Phase Signal Simulation Analysis for Precise Velocity and Position Determination, Proceedings of ION NTM 97, Santa Monica, CA, 1997.

Cohen, C. E., Altitude determination, in Global Positioning System: Theory and Applications, Vol. II, 18 pp., edited by Parkinson and Spilker, 1996.

Euler, H. J. and H. Landau, Fast GPS ambiguity resolution on-the-fly for real-time applications, Proceedings of 6th INt. Geod. Symp. on satellite Positioning. Columbus, Ohio, 17-20, 1992.

Goad, C. and B. Remondi, Initial relative positioning results using the global positioning system, Bulletin Geodesique, 58, 193-210, 1984.

Han, S. and C. Rizos, On-The-Fly Ambiguity Resolution for Long Range GPS Kinematic Positioning, IAG Symposia 115, 5 pp., edited by Beutler et al., 1995.

Han, S. and C. Rizos, Comparing GPS Ambiguity Resolution Techniques, GPS World, Oct. 1997, 54-61, 1997.

Hofmann-Wellenhof, B., H. Lichtenegger, and J. Collins, GPS Theory and Practice, 4-th edition, Springer-Verlag, Wien, 1997.

Jensen, A., Influences of reference coordinates on precise static/kinematic GPS positioning, 1999 (in preparation).

Klobuchar, J. A., Ionospheric Effects on GPS, in Chapter 12 of Global Positioning System: Theory and Applications, Vol. I, 30 pp., edited by B. W. Parkinson and J. J. Spilker, 1996.

Merbart, L., Ambiguity Resolution Techniques in Geodetic and Geodynamic Applications of the Global Positioning System, Dissertation, Philosophisch-naturwissenschaftlichen Fakultaet der Universitaet Bern, 1995

Ramatschi, M., Untersuchung von Vertikal-bewegungen durch Meeresgezeitenauflasten an Referenz-stationen auf Groenland, Dissertation, Technische Universitaet Clausthal, 1998.

Schwarz, K. P., M. E. Cannon, and R. V. C. Wong, A comparison of GPS kinematic models for the determination of position and velocity along a trajectory, Manuscripta Geodaetica, 14, 345-353, 1989.

Shfaqat, K. A., Ocean loading tide effects on GPS positioning, MSc. thesis, Copenhagen University, 78 pp., 1999.

Syndergaard, S., Retrieval Analysis and Methodologies in Atmospheric Limb Sounding Using the GNSS Radio Occultation Technique, Dissertation, Niel Bohr Institute for Astronomy, Physics and Geophysics, Faculty of Science, University of Copenhagen, 1999.

Teunissen, P. J. G., The least-squares ambiguity decorrelation adjustment: a method for fast GPS integer ambiguity estimation, J. Geod., 70, 65-82, 1995.

Wang, G., Z. Chen, W. Chen, and G. Xu, The Principle of the GPS Precise Positioning System, 343 pp., Surveying Publishing House, Beijing, China, 1988.

$\mathrm{Xu}$, G., L. Bastos, and L. Timmen, GPS Kinematic Positioning in AGMASCO Campaigns-Strategic Goals and Numerical Results, Proceedings of ION GPS-97 Conference in Kansas City (USA), 1173-1183, 1997.

$\mathrm{Xu}$, G., P. Schwintzer, and Ch. Reigber, KSGSoft (Kinematic/Static GPS Software)—Software User Manual, Scientific Technical Report 19/1998, GeoForschungsZentrum Potsdam, Germany, 1998.

$\mathrm{Xu}, \mathrm{G}$. and P. Knudsen, Earth tide effects on kinematic/static GPS posi- 
tioning in Denmark and Greenland, Physics and Chemistry of the Earth, 25(4), 409-414, 2000.

$\mathrm{Xu}, \mathrm{G}$. , Integer Ambiguity Search in Coordinate and Ambiguity Domains, paper presented at IUGG99 IAG Symposium, Birmingham, J. Geod., 1999a (submitted).

$\mathrm{Xu}, \mathrm{G}$. , Ionosphere-free ambiguity fixing, paper presented in CHAMP in- ternal meeting and prepared publication, $1999 \mathrm{~b}$.

$\mathrm{Xu}, \mathrm{G}$., On the general ambiguity search criterion, paper prepared for publication, J. Geod., 2000 (submitted).

G. Xu (e-mail: xu@gfz-potsdam.de) 